\title{
A New Multipath Routing Approach for Energy Efficiency in Wireless Sensor Networks
}

\author{
Saira Banu \\ Department of electronics and \\ Communication systems \\ Karpagam University, Coimbatore
}

\author{
R.Dhanasekaran, PhD. \\ Director, Dept. of EEE \\ Syed Ammal Engineering College, \\ Ramanathapuram
}

\begin{abstract}
In Wireless Sensor Networks (WSNs), sensors are organized randomly. Routing in the wireless sensor networks is a demanding assignment. This assignment may lead to a number of routing protocols which effectively use the limited resources available at the sensor nodes. So all the routing protocols will attempt to find the optimal energy path. In order to determined the alternative path quickly, there is need of reduction of energy path and time. Here We have proposed the New Multipath Routing Approach (NMRA) for increasing the energy efficiency in WSNs. It consists of three phases. In first phase, the multipath routing is constructed. In second phase, the optimal energy path is established. In third phase, the energy consumption model is developed. By simulation results, the proposed NMRA achieves better delivery ratio, improved network lifetime, less delay and energy consumption in terms of mobility, time and number of nodes than the existing scheme SByaoGG.
\end{abstract}

\section{General Terms}

New Multipath Routing Approach

\section{Keywords}

WSN, NMRA, Multipath routing, optimum energy path, network lifetime, delay, energy consumption, throughput and delivery ratio

\section{INTRODUCTION}

attention in recent years due to the advances made in wireless communication, information technologies and electronics field. It is an -In situll sensing technology where tiny, autonomous and compact devices called sensor nodes or motes deployed in a remote area to detect phenomena, collect and process data and transmit sensed information to users. The development of lowcost, low-power, a multifunctional sensor has received increasing attention from various industries. Sensor nodes or motes in WSNs are small sized and are capable of sensing, gathering and processing data while communicating with other connected nodes in the network, via radio frequency (RF) channel.

WSN term can be broadly sensed as devices range from laptops, PDAs or mobile phones to very tiny and simple sensing devices. At present, most available wireless sensor devices are considerably constrained in terms of computational power, memory, efficiency and communication capabilities due to economic and technology reasons. That's why most of the research on WSNs has concentrated on the design of energy and computationally efficient algorithms and protocols, and the application domain has been confined to simple data- oriented monitoring and reporting applications. WSNs nodes are battery powered which are deployed to perform a specific task for a long period of time, even years. If WSNs nodes are more powerful or mains-powered devices in the vicinity, it is beneficial to utilize their computation and communication resources for complex algorithms and as gateways to other networks. New network architectures with heterogeneous devices and expected advances in technology are eliminating current limitations and expanding the spectrum of possible applications for WSNs considerably.

\subsection{Design goals of WSNs}

Based on the application, different architecture, goals and constraints have been considered for WSNs. As discussed in [18], the following design goals are given.

\subsubsection{Energy Considerations}

During the creation of an infrastructure, the process of setting up the routes is greatly influenced by energy considerations. Since the transmission power of a wireless radio is proportional to distance squared or even higher order in the presence of obstacles, multihop routing will consume less energy than direct communication. However, multi-hop routing introduces significant overhead for topology management and medium access control. Direct routing would perform well enough if all the nodes were very close to the sink. Most of the time sensors are scattered randomly over an area of interest and multi-hop routing becomes unavoidable.

\subsubsection{Node deployment}

Node deployment in WSN is application dependent and affects the performance of the routing protocol. The deployment can be either deterministic or randomized. In deterministic deployment, the sensors are manually placed and data is routed through predetermined paths; but in random node deployment, the sensor nodes are scattered randomly creating an infrastructure in an ad hoc manner. Hence, random deployment raises several issues as coverage, optimal clustering etc. which need to be addressed.

\subsubsection{Energy consumption without losing accuracy}

Sensor nodes can use up their limited supply of energy performing computations and transmitting information in a wireless environment. As such, energy conserving forms of communication and computation are essential. Sensor node lifetime shows a strong dependence on the battery lifetime. In a multihop WSN, each node plays a dual role as data sender and data router. The malfunctioning of some sensor nodes due to power failure can cause significant topological changes and might require rerouting of packets and reorganization of the network.

\subsubsection{Fault Tolerance}

Some sensor nodes may fail or be blocked due to lack of power, physical damage, or environmental interference. The failure of sensor nodes should not affect the overall task of the sensor network. If many nodes fail, MAC and routing protocols must accommodate formation of new links and routes to the data 
collection base stations. This may require actively adjusting transmit powers and signalling rates on the existing links to reduce energy consumption, or rerouting packets through regions of the network where more energy is available. Therefore, multiple levels of redundancy may be needed in a fault-tolerant sensor network

\subsubsection{Quality of Service}

In some applications, data should be delivered within a certain period of time from the moment it is sensed; otherwise the data will be useless. Therefore bounded latency for data delivery is another condition for time-constrained applications. However, in many applications, conservation of energy, which is directly related to network lifetime, is considered relatively more important than the quality of data sent. As the energy gets depleted, the network may be required to reduce the quality of the results in order to reduce the energy dissipation in the nodes and hence lengthen the total network lifetime. Hence, energyaware routing protocols are required to capture this requirement.

\subsubsection{Data Aggregation/Fusion}

Since sensor nodes might generate significant redundant data, similar packets from multiple nodes can be aggregated so that the number of transmissions would be reduced. Data aggregation is the combination of data from different sources by using functions such as suppression (eliminating duplicates), min, max and average. In some network architectures, all aggregation functions are assigned to more powerful and specialized nodes. Data aggregation is also feasible through signal processing techniques. In that case, it is referred as data fusion where a node is capable of producing a more accurate signal by reducing the noise.

\section{RELATED WORK}

Hannes Frey et.al [1] proposed new multicast generalization with fast recovery mechanism to achieve a delivery of multicast message, to improve energy efficiency and path to the destination messages. The proposed solution is based on minimum spanning tree (MST) which requires information only on single hop neighbors. A message replication occurs when the MST spanning the current node and the set of destinations has multiple edges originated at the current node. Destinations spanned by these edges are grouped together, and for each of these subsets the best neighbor is selected as the next hop. This selection is based on a cost over progress metric, where the progress is approximated by subtracting the weight of the MST over a given neighbor and the subset of destinations to the weight of the MST over the current node and the subset of destinations.

Tomas Johansson et.al [] proposed a Localized AreaSpanning Tree (LAST) for wireless short-range sensor networks which comprises of selecting child nodes and target points. They consider a problem of building a forwarding tree for multicast and convergecast traffic in short-range wireless sensor networks. Interference awareness and energy efficiency are the major design objectives for WSN protocols in order to maximize the network lifetime. When creating the tree, the protocol jointly optimized the energy cost and the interference imposed by the structure.

S.G.Santhi and K.Venkatachalapathy [] explored a technique called an ant based multiple cluster tree routing for 802.15.4 sensor networks. In this approach, a node was randomly selected among the available nodes as the PAN coordinator. The PAN coordinator utilized the swarm intelligence based ant colony optimization technique to select the nodes within the transmission range for cluster formation which corresponds to the trees. In order to achieve the diverse topologies of different trees, a proper parent is selected based on the link quality index. Further, each node selects the tree with minimum cost as the main routing tree adaptable to fault free multimedia traffic. The QoS based routing was utilized for cluster based multi tree topology using ant agents.

Mojtaba Khayat [] developed a source-specific multicast protocol for wireless mesh network, which has many application in, multimedia, radio and TV multicasting and distance learning. They have used core-based approach to construct minimum cost tree (MCT) among member nodes and optimized this tree for multiple metrics by applying ant colony optimization metaphor. In this work they have used weighted sum of two network metric; delay and number of non-forwarding node on new paths to calculate reinforcement value, but we can extend these metric to include bandwidth, jitter or even security metrics.

Rathna. R et.al [2] explored how to improve the energy efficiency in WSN through the scheduling and routing. In scheduling scenario, on regular time interval all the nodes will be either in sleep mode or active mode. The proposed algorithm is completely TDMA based. It helps to reduce the energy consumption by reducing the number of times. A node has to wake up, during a time slot, to be in active mode. The underlying concept is efficient usage of energy. It has been proved also. The time delay is also reduced to a small extent.

Meenakshi Diwakar And Sushil Kumar [3] developed the protocol called Energy-Efficient Level Based Clustering Routing Protocol (EELCBRP) to improve the lifetime of the networks. They have considered the residual energy of each node and distance from the BS of nodes as the principle of cluster head election. The mathematical formulae for election the cluster head is provided. The simulation results of energy consumption of cluster heads, numbers of clusters and network lifetime are provided. They observed that the energy consumed by $\mathrm{CHs}$ for each round in EELBCRP is much lower than that in LEACH.

S.Barani and Dr.C.Gomathy [4] introduced energy aware routing algorithm for wireless sensor networks. Protocol is based on fuzzy logic computation reduces power consumption by the ratio of 10 when compared to classical routing protocol. Proposed protocol is implemented in two phases, first phase being computation of neighbor node and updating of routing table and route establishment is done in second phase. Consumption of energy is considerably reduced on properly designed protocol.

Lei Wang et.al [5] developed networks with network codingbased multipath routing for energy efficiency. The proposed multipath model is braided multipath model, and the used network coding scheme is random linear network coding. Braided multiple paths are established to the sink node for each source node, and the packets encoded at source nodes are transmitted on the braided multipath network. Then, the intermediate nodes are re-encoded the received packets and forward the new packets to next cluster. Finally, the sink node decodes the packets received from different paths and recovers the original data. When network coding is combined with multipath routing, the number of required routes and the total times of transmission in sensor networks are reduced, which leads to energy consumption of proposed multipath routing is lower than that of traditional multipath routing. 
Xufei Mao et.al [6] explored energy efficient opportunistic routing in wireless sensor networks which selects the prioritizing forwarder list to minimize energy consumptions by all nodes. In opportunistic routing, in order to improve the network throughput, it is allowed nodes that overhear the transmission and closer to the destination to participate in forwarding the packet. The nodes in forwarder list are prioritized and the lower priority forwarder will discard the packet if the packet has been forwarded by a higher priority forwarder. The Major challenging problem is to select and prioritize forwarder list such that a certain network performance is optimized.

Nidhi Batra et.al [7] proposed the optimized routing protocol which is focussed on energy wastage. One of the upcoming research area of it is its power consumption which in turn depends on energy wastage of nodes. The energy efficient shortest path routing algorithm proposed includes Medium Access Control (MAC) protocols and routing protocols of WSN. It also includes the concept of Ant Colony Optimization (ACO) for getting the shortest path between sender and receiver. Here the proposed algorithm is cost effective.

Zhenhua $\mathrm{Yu}$ et.al [8] introduced the a knowledge-based inference approach using fuzzy Petri nets which is employed to select cluster heads and then the fuzzy reasoning mechanism is used to compute the degree of reliability in the route sprouting tree from cluster heads to the base station. Finally, the most reliable route among the cluster heads can be constructed. The algorithm is not only balanced the energy load of each node but also provided the global reliability for the whole network. The proposed algorithm effectively prolonged the network lifetime and reduces the energy consumption.

Manjunath et.al [9] proposed the routing scheme that assures high accuracy and significantly reduces data transmission costs in WSN with faults. A number of network topologies are randomly used for routing sensor readings to the base station. Because every sensor node is connected to each other with a single path, redundant transmissions are not incurred. It can reduce unnecessary transmissions and guarantee final sensor readings with high accuracy.

Gaurav Bathla et.al [10] developed proposed algorithm with data aggregation $\&$ fusion which is used to minimize reduction in system energy by first generating Minimum Spanning Tree between all sensor nodes so as to minimize their transmission energy with in network and after that a node of highest energy among the top tier will transmit the aggregated data of whole network to base station. They have kept network topology same till any node of network dies another highest energy node from top most rank tier is chosen to communicate with Base Station.

Yuping Dong et.al [11] proposed energy efficient routing algorithm for WSN. In this algorithm, they have divided the sensor nodes into several scheduling sets and let them work alternatively. In this way, the sensors do not have to be active all the time which saves a lot of energy. When choosing the next sensor to forward the information to, they considered both the distance from the base station to the sensor and its current energy level. So the network power consumption will be distributed among the sensors. When the network does not have enough sensors that have sufficient energy to run, it generates new scheduling sets automatically.

Rakesh Kumar Yadav et.al [12] compared some of the data dissemination protocols for WSN. Due to many constraints of nodes in wireless sensor networks, a variety of data dissemination protocols have been developed for data gathering in wireless sensor networks. Sensors extract useful information from environment; this information has to be routed through several intermediate nodes to reach the destination. Effective information dissemination is one of the most important tasks in sensor networks. Directed Diffusion is a communication paradigm for information dissemination in WSNs based on data centric routing.

Sasmita Sahoo and Biswa Mohan Acharya [13] proposed the energy efficient routing protocol in Wireless Sensor Network. Routing is a serious issue in WSN due to the use of computationally-constrained and resource constrained microsensors. Once the sensor nodes are deployed replacement is not feasible. Hence, energy efficiency is a key design issue to improve the life span of the network. Since the network consists of low-cost nodes with limited battery power, it is a challenging task to design an efficient routing scheme that can offer good performance in energy efficiency, and long network lifetimes.

Golam Rashed et.al [14] introduced a scheme to combine clustering strategy with chain routing algorithm for satisfy both energy and stable period constrains under heterogeneous environment in WSNs.

Imanishimwe Jean de Dieu et.al [15] explored a new energy efficient secure path algorithm for wireless sensor networks to ensure the secure sensed data in a balanced energy network backbone. The proposed algorithm overcomes the limitations caused by the symmetric cryptographic algorithms for securing data in sensor networks. They have achieved the authenticity and integrity on the actual sensed data within the energy efficient infrastructure. The key contribution is to ensure secure transmission which results prolonging network lifetime via a suboptimal energy-efficient and balancing routing algorithm.

The paper is organized as follows. The Section 1 describes introduction about WSNs, design goals and issues. Section 2 deals with the previous work which is related to the multipath algorithms. Section 3 is devoted for the implementation of proposed algorithm. Section 4 describes the performance analysis and the last section concludes the work.

\section{IMPLEMENTATION OF PROPOSED ALGORITHM}

Our proposed Multipath routing scheme consists of 3 steps like multipath construction phase, Maintenance of optimal energy path and Energy consumption model to improve the energy efficiency in Sensor networks.

\subsection{Multipath Construction Phase}

- multipath path construction phase to create a set of neighbors that is the address of all nodes that are able to transmit data from the source.

- In this process route request control messages are exchanged between the nodes. Each sensor node broadcasts the route request packet once and maintains its own routing table. When sensor node broadcasts a data packet to the known neighboring nodes. It does not maintain the whole routing information. It is necessary to store the routing information and it reduces the overhead of sensor node while it requires the proactive protocol.

- The multipath routing protocol has to calculate some information to record in the routing table of sensor node, the energy expense is less than transmit and receive. Furthermore, it supports multipath data forwarding, not 
using the fixed path. So the energy consumption will be distributed and the lifetime of network is prolonged.

- The major activities in this phase are routing path formation for each node and neighbor table creation. The sink node broadcasts the route request packet to discover the one hop nodes / level 1 nodes, the nodes which are receiving them first.

- Route Request control messages are used to identify nodes in different levels. After a route request message is sent by sink node, the hop count records how many hops it has travelled from the sink.

\section{- The Format of Route Request Control Message}

The Source ID contains the node ID of the message destination; SeqNumber field is a packet sequence. The HopCount field is the number of hops from the sink node which is used to identify nodes in different levels, nodes that can receive the radio signal of sink are defined as onehop / level 1 nodes, Energy threshold field provides the minimum required energy level for a node to be selected for data transmission, Signal Strength threshold to indicate the minimum distance the node has to be located in order to receive all the data's transmitted to that node and Sink ID indicates the ID of the sink which broadcasts the route request packet.

- The hop count field is increased by one each time when a node receives the route request message. When receiving a route request, a node considers itself in level $\mathrm{N}$ if the hop count is $\mathrm{N}$. If a smaller hop count like N-1 is received later from a route request with the same sequence number, as the current remembered, the node updates its level according to the new hop count. Smaller hop count nodes constantly use less energy than others.

- Once the HopCount field is incremented, it is compared with the nodes hop value. If HopCount field is smaller than node's hop value, route request message is processed or otherwise drops the message. The corresponding node is then responsible to rebroadcast the route request message to its neighbors.

- The nodes which process the route request message are liable to form a path to the destination (i.e.) source. Unlike other energy-aware routing protocols, which attempt to find minimum-energy-cost paths, this protocol provides energy-sufficient paths instead.

- A special flooding mechanism is adopted in the routing path formation. When an intermediate node receives the route request message, it does not forward the message to its neighbors immediately.

- Before sending the message out, several things are done. The intermediate node first checks the hop value if it is found to be lesser, it starts checking nodes available energy. If the available energy is less than operation energy (e.g., twice the packet transmission energy), that indicates that the node has no more energy to take more transmission jobs, the node simply discards the received request.

- If the node has sufficient energy then the node measures the Received Signal Strength (RSS) which is the strength of the route request message. This received signal strength measurement is indicated by the nodes Radio Signal Strength Indicator (RSSI) value. RSS is based on the principal that a radio signal between a sender and a receiver attenuates with an increase in distance.

\subsection{Maintenance of Optimal Energy Path}

We use the concept of optimal energy path to estimate minimum energy consumption. The main aim is to maintain the data packet flow in the wireless sensor network unobstructed.

The definition of the sensor network model is given as follows. Let us assume a given network $\mathbf{N}(\Psi, \Theta)$ is composed of $\Psi$ and $\Theta$, where $\Psi$ is the set of nodes and $\Theta$ is the set of links. While taking the feature of multi-hop transmission, sensor network could have many paths from source node $S$ to destination node $D$. Therefore, let $\Pi(\mathrm{S}, \mathrm{D})$ denote the set of all possible paths starting from $\mathrm{S}$ to $D$. According to these definitions, it is known that $\boldsymbol{\Pi}(s, d)$ is the subset of $\boldsymbol{\Lambda}$. Let $\boldsymbol{\pi}$ represent a generic path, and $\boldsymbol{\pi}_{i(S, D)}$ represents $i$-th path in a journey from source node $s$ to destination node $d$. Also, we let $\Phi(\boldsymbol{\pi})$ be a generic cost function associated to a designated path $\boldsymbol{\pi}$. $\Phi(\boldsymbol{\pi})$ can be the delay time $\theta(\boldsymbol{\pi})$ for a packet transfers through a path $\pi$, or the number of hops $\varepsilon(\pi)$, even a hybrid function combined both of them. For two or more paths possessed the same cost, we consider them as $\Phi$-equivalent.

Since each node in the WSN functions as a router that works independently, several connection matrices are defined and stored in each node to help performing the energy efficient routing function:

If we derive the connection matrix as $T s=[t s, d]$, where $T s$ is an array that registers all outgoing links of node $s$, and $t s, d$ determines whether node $s$ has an outgoing link connected to node $d$ or not, 1 represents connected, 0 represents disconnected. Let $B s, d$ denotes the bandwidth utility ratio of link from node $s$ to node $d . p s, d f$ that determines the probability table for deciding the next node of a packet transferring from node $s$ to its final destination node $d f, . p F, d f, \mathrm{~K}$ corresponds to the probability for the packet transferred to node $j$. Note that $\Sigma j(p F, d f, K)=1$. Also, suppose that Efull is the initial energy on each node and $E_{k}$ is the remaining energy on node $K$. With the above-given notations, the weight of choosing node $j$ as the next node for transferring the packet while the packet is queued at node $s$ is,

$w(F, K, d f)=t_{F, K} \cdot\left[p_{F, d f, k}\right]^{V_{1}} \cdot\left[1-B_{K, F}\right]^{V_{2}} \cdot\left[E_{f} / E_{f u l l}\right]^{V_{3}} \cdot\left[E_{f, r e m}\right]^{V_{4}}$

where $\mathrm{V}_{1}, \mathrm{~V}_{2}, \mathrm{~V}_{3}$ and $\mathrm{V}_{4}$ are weighting factors that regulate the importance of probability matrix $(\mathrm{pF}, \mathrm{df})$, bandwidth utility ratio $\left(\mathrm{B}_{\mathrm{F}, \mathrm{K}}\right)$, and remaining energy ratio $\left(\mathrm{E}_{\mathrm{f}} / \mathrm{E}_{\text {full }}\right)$ during routing process, respectively.

Equation functions like a firing strength for the routing algorithm to send a packet from node $\mathrm{S}$ to node $\mathrm{f}$. The values of $\mathrm{V}_{1}, \mathrm{~V}_{2}, \mathrm{~V}_{3}$ and $\mathrm{V}_{3}$ are set to 1 in most cases. There is no systematic approach to obtain the optimal values of these parameters since it involves too many human factors. The probability of choosing node $\mathrm{j}$ as next node of the packet can be defined as follow

$\varsigma_{s, f}=\frac{w(f, k, d f)}{\sum_{k} w(f, h, d f)}$

The load-balance feature of the proposed scheme can be exploited using Equation (2). Eq. (2) is a normalized firing strength to send a packet from node $\mathrm{f}$ to node $\mathrm{k}$. This equation contains the terms of bandwidth utility ratio $\left(\mathrm{B}_{\mathrm{f}, \mathrm{k}}\right)$ and remaining energy ratio $\left(\mathrm{E}_{\mathrm{f}} / \mathrm{E}_{\text {full }}\right)$. In the investigation, once the packets 
reaches to its final destination node through a path $\pi \mathrm{i}(\mathrm{S}, \mathrm{D})$, the cost function of the path can be defined as follow

$\varphi\left(\Pi_{i}(s, d)\right)=\theta\left(\Pi_{i}(s, d)\right)$

The probability matrix ps, df according to the following equation

$$
p_{s, d f, k}=\rho \cdot p_{s, d f, k}+\Delta p_{s, d f, k} \cdot \forall(s, d f, k)_{(4)}
$$

where $\rho$ is the learning rate. In order to keep the probability matrix ps, df normalized, every time after $\mathrm{ps,df}$ has been updated by Equation (4). We have to perform normalization on $\mathrm{p}_{\mathrm{s}, \mathrm{df}}$ using,

$$
p_{s, d f, k}=\frac{P_{s, d f, k}}{\sum_{\forall h} p_{s, d f, h}}
$$

These parameter updating procedures will be executed once a packet reaches its destination node.

\subsection{Packet Loss in WSNs}

Packet loss due to congestion is a fundamental problem in managed sensor networks. A queue forms in a buffer until the router is able to transmit them on their way. In this thesis, it is considered that buffer overflow takes place when a queue exceeds its buffer limit i.e tail-drop queues. Random Early Discard (RED) mechanisms cause packet drop before the buffer is full.

\subsection{Energy Consumption Model}

The title (Helvetica 18-point bold), authors' names (Helvetica 12-point) and affiliations (Helvetica 10-point) run across the full width of the page - one column wide. We also recommend email address (Helvetica 12-point). See the top of this page for three addresses. If only one address is needed, center all address text. For two addresses, use two centered tabs, and so on. For three authors, you may have to improvise.

\section{PERFORMANCE ANALYSIS}

We use Network Simulator (NS2) to simulate our proposed algorithm. Network Simulator-2(NS2) is used in this work for simulation.NS2 is one of the best simulation tools available for Wireless sensor Networks. We can easily implement the designed protocols either by using the otcl coding or by writing the $\mathrm{C}++$ Program. In either way, the tool helps to prove our theory analytically.

In our simulation, 200 mobile nodes move in a 1200 meter $\mathrm{x}$ 1200 meter square region for 60 seconds simulation time. All nodes have the same transmission range of 250 meters. Our simulation settings and parameters are summarized in table 2 .

Table2. Simulation settings and parameters of proposed algorithm.

\begin{tabular}{|l|l|}
\hline No. of Nodes & 200 \\
\hline Area Size & $1200 \mathrm{X} 1200$ \\
\hline Mac & 802.11 \\
\hline Radio Range & $250 \mathrm{~m}$ \\
\hline Simulation Time & $60 \mathrm{sec}$ \\
\hline Traffic Source & CBR \\
\hline Packet Size & $512 \mathrm{bytes}$ \\
\hline Mobility Model & Random Way Point \\
\hline Transmitter Amplifier & $150 \mathrm{pJ} / \mathrm{bit} / \mathrm{m}^{2}$ \\
\hline
\end{tabular}

\begin{tabular}{|l|l|}
\hline Package rate & $5 \mathrm{pkt} / \mathrm{s}$ \\
\hline Protocol & DSR \\
\hline
\end{tabular}

\subsection{Performance Metrics}

We evaluate mainly the performance according to the following metrics.

End-to-end delay: The end-to-end-delay is averaged over all surviving data packets from the sources to the destinations.

Data Availability Ratio: It is defined as the making the copies of data items which shared by several users in a particular point of time.

Throughput: It is defined as the number of packets received at a particular point of time

The simulation results are presented in the next part. We compare our proposed algorithm (NMRA) with SBYaoGG [18] in presence of energy consumption.

Figure 1 shows the results of average residual energy for varying the time from 10 to 50 . From the results, we can see that NMRA scheme has minimal energy consumption than the SBYaoGG scheme

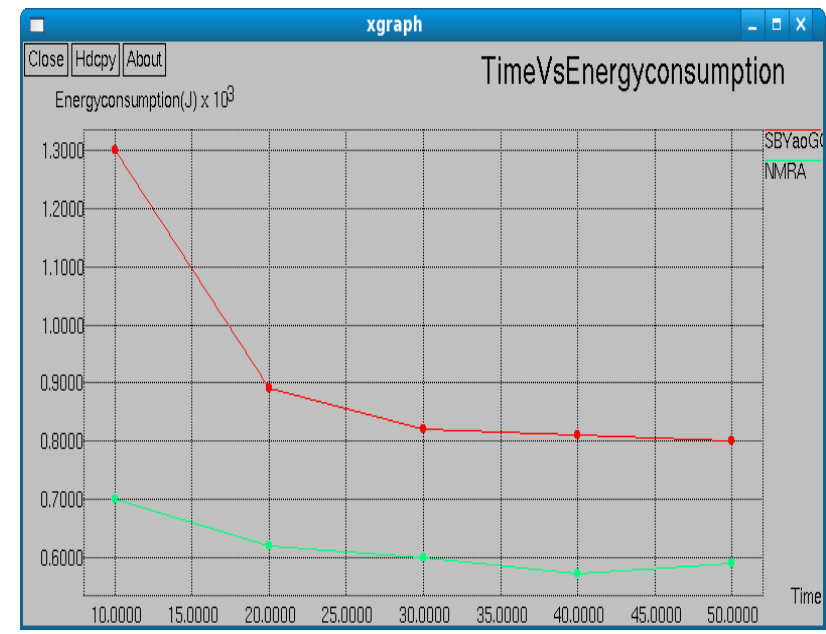

Fig. 1. Time Vs Energy consumption

Fig. 2. presents the network lifetime comparison for NMRA, SBYaoGG. It is clearly seen that number of epochs consumed by NMRA is high compared to SBYaoGG.

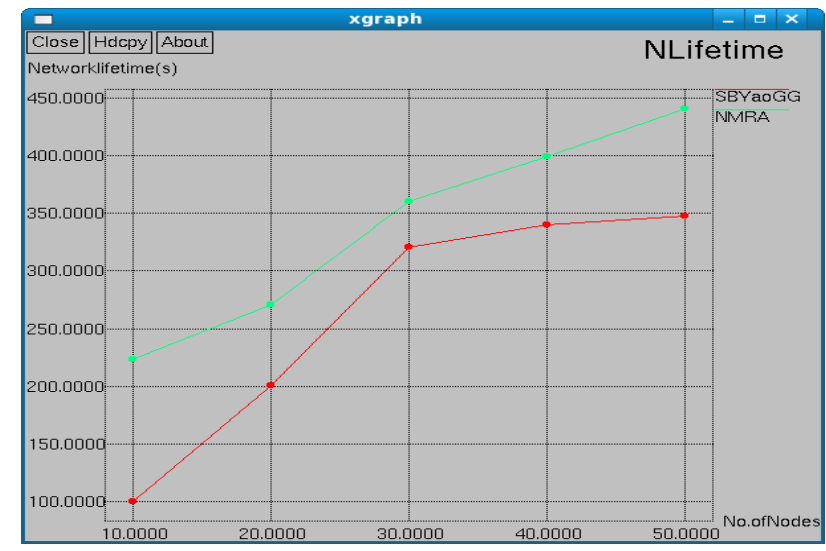

Fig. 2. Increasing the network lifetime 
Fig. 3, presents the comparison of data availability. It is clearly shown that the data availability ratio of NMRA is higher than the SBYaoGG

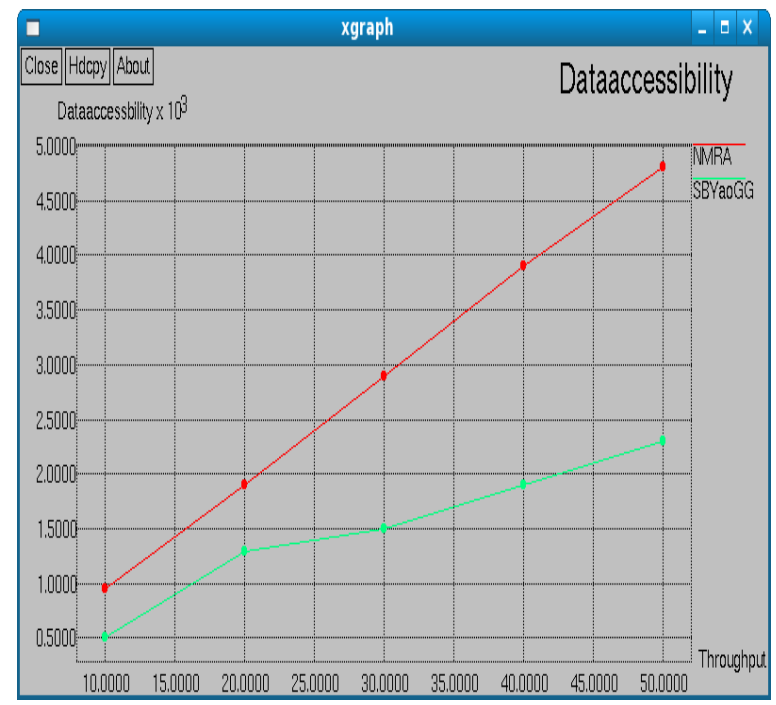

Fig. 3. Throughput Vs Data Availability

Figure 4 shows the results of Time Vs End to end delay. From the results, we can see that NMRA scheme has slightly lower delay than the SBYaoGG scheme because of authentication routines.

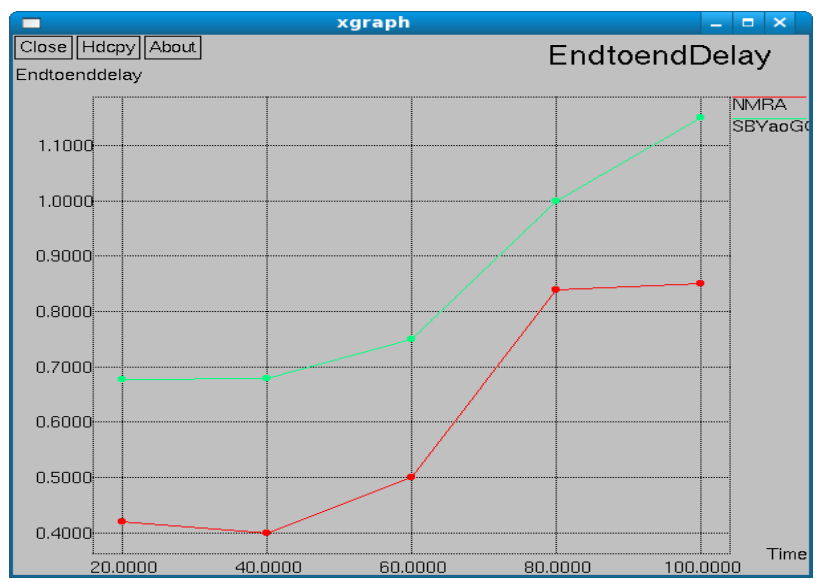

Fig. 4. Time Vs End to end delay

Fig. 5, presents the comparison of delay while varying the mobility from 5 to 25 . It is clearly shown that the delay of NMRA is lower than the SBYaoGG.

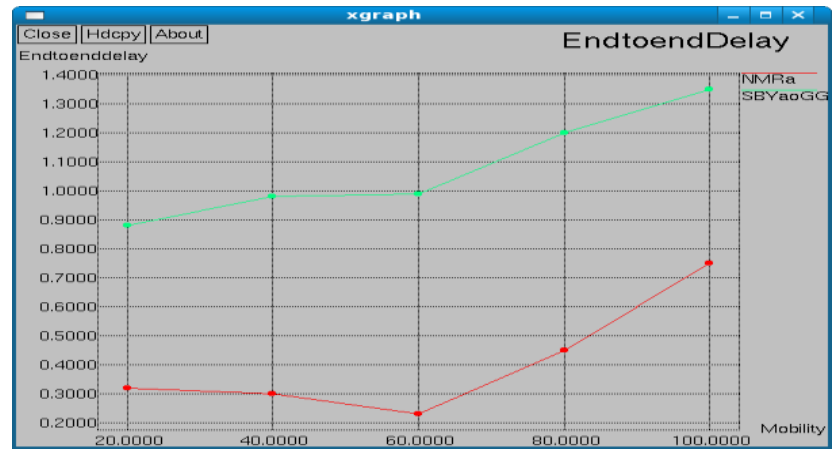

Fig. 5. Mobility Vs Delay

\section{Conclusion}

In WSNs, the best route is being determined by choosing efficient strategy to forward the data to the base station. Due to that, the node consumes more energy unnecessarily. In this paper, we have developed a New Multipath Routing Approach which attains energy model, maintenance of optimal energy path, multipath construction phase to make a correct balance between network life time, energy consumption and throughput to the sensor nodes. In the first phase of the scheme, construction of multipath is implemented. In second phase, the optimal energy path is maintained. In third phase, residual energy consumption is increased using energy model. It uses following factors called distance, residual energy, mobility factor, mobility factor and data correlation to favor packet forwarding by maintaining high residual energy consumption for each node. We have demonstrated the energy estimation of each node. By simulation results we have shown that the NMRA achieves good throughput, high network lifetime, high residual energy while attaining low delay than the existing schemes SBYaoGG while varying the number of nodes, time, node throughput and mobility.

\section{REFERENCES}

[1] V.A.Amala Shiny \& V.Nagarajan, "Energy Efficient Routing Protocol for Mobile Wireless Sensor Network", International Journal of Computer Applications (0975 8887), Volume 43- No.21, April 2012, pp.1-5.

[2] Rathna. R And Sivasubramanian. A, "Improving Energy Efficiency In Wireless Sensor Networks Through Scheduling And Routing", International Journal Of Advanced Smart Sensor Network Systems ( IJASSN ), Vol 2, No.1, January 2012, pp.21-27.

[3] Meenakshi Diwakar And Sushil Kumar, "An Energy Efficient Level Based Clustering Routing Protocol for Wireless Sensor Networks", International Journal Of Advanced Smart Sensor Network Systems ( IJASSN ), Vol 2, No.2, April 2012, pp.55-65.

[4] S.Barani and Dr.C.Gomathy, "Energy Aware Routing Algorithm for Wireless Sensor Network", Indian Journal of Computer Science and Engineering (IJCSE), Vol. 2 No. 6 Dec 2011-Jan 2012, pp.850-861.

[5] Lei Wang, Yuwang Yang and Wei Zhao, "Network coding-based multipath routing for energy efficiency in wireless sensor networks", EURASIP Journal on Wireless Communications and Networking, 2012, pp.1-15.

[6] Xufei Mao, Shaojie Tang, Xiahua Xu, Xiang-Yang Li, Huadong Ma, "Energy Efficient Opportunistic Routing in Wireless Sensor Networks", IEEE Transactions On Parallel And Distributed Systems, 2011, pp.1-14.

[7] Nidhi Batra, Anuj Jain, Surender Dhiman, “An Optimized Energy Efficient Routing Algorithm For Wireless Sensor Network", International Journal Of Innovative Technology \& Creative Engineering (ISSN: 2045-8711), Vol.1 No.5, May 2011, pp.41-45.

[8] Zhenhua Yu, , Xiao Fu, Yuanli Cai and Mehmet C. Vuran, “ A Reliable Energy-Efficient Multi-Level Routing Algorithm for Wireless Sensor Networks Using Fuzzy Petri Nets", Sensors 2011, 11,pp. 3381-3400. 
[9] Manjunath K.G. , Dr. Jaisankar N. and Pradeep M , "An Efficient Routing Scheme for Wireless Sensor Networks", International Journal of Computer Science and Information Technologies, Vol. 2 (4), 2011, pp.1798-1801

[10] Gaurav Bathla and Gulista Khan, "Energy- efficient Routing Protocol for Homogeneous Wireless Sensor Networks", International Journal on Cloud Computing: Services and Architecture (IJCCSA), Vol. 1, No. 1, May 2011, pp.12-20.

[11] Yuping Dong , Hwa Chang, Zhongjian Zou and Sai Tang, "An Energy Conserving Routing Algorithm for Wireless Sensor Networks", International Journal of Future Generation Communication and Networking, Vol. 4, No. 1, March 2011, pp.39-54.

[12] Rakesh Kumar Yadav, Kusum Gupta, Ajay Singh Yadav, "A Comparison of Data Dissemination Protocols for Wireless Sensor Networks", International Journal of Scientific \& Engineering Research Volume 2, Issue 7, July2011, pp.1-3.

[13] Sasmita Sahoo, Biswa Mohan Acharya, "Energy Efficient Routing Protocol In Wireless Sensor Network", International Journal of Engineering Science and Technology, Vol. 3 No. 7 July 2011, pp.6084-6089.

[14] Md. Golam Rashed, M. Hasnat Kabir and Shaikh Enayet Ullah, "WEP: An Energy Efficient Protocol For Cluster Based Heterogeneous Wireless Sensor Network", International Journal of Distributed and Parallel Systems (IJDPS) Vol.2, No.2, March 2011, pp.54-60.

[15] Imanishimwe Jean de Dieu, Nyirabahizi Assouma, Maniraguha Muhamad,Wang Jin, and Sungyoung Lee, "Energy-Efficient Secure Path Algorithm for Wireless Sensor Networks", International Journal of Distributed Sensor Networks, 2012, pp.1-15.

[16] Kajal V Shukla and Priyanka K Shah, "A Survey on Energy Efficient Routing Techniques for Wireless Sensor
Networks", Journal of Information And Communication Technologies, Volume 2, Issue 1, January 2012, pp.29-35.

[17] Sanatan Mohanty, "Energy Efficient Routing Algorithms for Wireless Sensor Networks and Performance Evaluation of Quality of Service for IEEE 802.15.4 Networks", Thesis on Sensor Networks, 2010, pp.1-127.

[18] Tapiwa M. Chiwewe, and Gerhard P. Hancke, "A Distributed Topology Control Technique for Low Interference and Energy Efficiency in Wireless Sensor Networks", IEEE Transactions On Industrial Informatics, Vol. 8, No. 1, February 2012, pp.11-19.

\section{AUTHORS PROFILE}

Dr. R.Dhanasekaran received M.E in Power Electronics and drives from Anna University, Chennai and awarded Ph.D by Anna University, Chennai in 2007. He is currently the Director, Research in Department of Electrical \& Electronics Engineering, Syed Ammal Engineering College, Ramanathapuram. His research interest includes EMI, Electrical Engineering and Power Drives. He has about 13 years of teaching and research experience. He has published 39 papers in National and 7 in International conferences and also published 8 papers in international journals.

S.SairaBanu received M.Sc degree from RVS College of Arts and Science in 2002 , M.Phil degree from RVS College of Arts and Science in 2007 and pursuing the Ph.D. degree in Electronics from Bharathiar University. She is currently working as Assistant Professor in Department of Electronics and Communication Systems, Karpagam University. Her research interest includes Biomedical Instrumentation, Wireless Communication and Networking. She has about 10 years of teaching experience She has Presented 7 Papers in National Conferences. 